\title{
THE GENESIS OF THE ELECTROCARDIOGRAM OF RIGHT VENTRICULAR HYPERTROPHY
}

\author{
BY \\ M. McGREGOR \\ From the Institute of Cardiology, The National Heart Hospital, London
}

Received April 12, 1950

The electrocardiographic changes considered diagnostic of hypertrophy of the right ventricle are well known. They are characterized by a predominantly positive initial ventricular complex over the right chest, preceded by a small q wave or $\mathrm{r}-\mathrm{s}$ waves, while over the left chest a predominantly negative complex preceded by a small $r$ wave is found.* The transition point between the $\mathrm{qR}$ (or rR) and the rS patterns lies usually in the sternal region anteriorly (Fig. 4), but may frequently be somewhat to the right of the sternum in position V3R (Fig. 7). In some cases in which $R-S$ waves of equal size occur across the whole left præcordium a sharp transition point between $\mathrm{rS}$ and $\mathrm{qR}$ or $\mathrm{rR}$ complexes can be found by taking leads from areas slightly more cranial than the standard positions (Fig. 6). The value and shortcomings of the standard leads in right ventricular hypertrophy are well known and have recently been reviewed by Myers, Klein, and Stofer (1948); they will not be discussed in this paper.

There would appear to be a substantial body of opinion which holds that these characteristic changes are due to an electrical predominance of the right ventricle as a result of its increased bulk, the normal potential differences between the right and left ventricles being reversed (Wilson et al., 1944). Thus, according to Goldberger (1944), leads facing the epicardial surface of the hypertrophied right ventricle show a positive or upright (qR) complex, while patterns derived from the left ventricular epicardium are mainly negative (rS) complexes, similar to those recorded over the right ventricle in health.

Consideration of the heart's position in such conditions as the tetralogy of Fallot without cardiac enlargment shows, however, that leads from the left side of the præcordium (e.g., 2-5) do not in fact face the epicardial surface of the left ventricle but the right (Fig. 2). This anomaly has been noted before (Kossman et al., 1948; Sokolow and Lyon, 1949). The right ventricle, when it hypertrophies, bulges forward and to the left so that the whole antero-lateral surface of the heart consists of right ventricular wall. This fact has been frequently confirmed during the cardiac catheterization of cases of the tetralogy of Fallot when the tip of the radio-opaque catheter can be seen on the X-ray screen to pass to the left lateral edge of the cardiac silhouette while still in the right ventricle. Furthermore, it is probable that leads from the right præcordium (e.g. C3R to C8R) do not face the right ventricle at all as the tricuspid valve can be demonstrated approximately in the midline of the body by angiocardiography.

The relationship of the patterns derived from the chest wall to the position of the underlying heart in health is illustrated in Fig. 1. As would be expected, electrodes facing the right ventricular epicardium record a right ventricular (rS) pattern while electrodes facing the left ventricle record a

* A predominantly positive complex preceded by a small $\mathrm{q}$ or $\mathrm{r}$ wave such as is seen in position V3R in Fig. 2 will, for the sake of brevity, be referred to as $\mathrm{qR}$ or $\mathrm{rR}$ pattern. Likewise, a predominantly negative complex preceded by a small $r$ wave such as the tracing V2 in Fig. 2 will be referred to as an rS pattern. 
Fig. 1.-Diagram and electrocardiogram for man (W) with carcinoma of the lung and a normal cardiovascular system. Diagram represents a schematic transverse section of the thorax through the region of the cardiac apex and the preoperative electrocardiogram. On the left are electrograms from the left auricular appendage (L.A.Appe.E.), the left ventricle (L.V.E.) and the right ventricle (R.V.E.). During the period in which these records were obtained the rhythm was nodal. Large $\mathbf{P}$ waves are apparent, superimposed on the ventricular complex in lead L.A.App.E. The standardization curve of the electrograms is also shown in the curve above them.

left ventricular (qR) pattern; the transition zone is in position V3. In the case of the tetralogy of Fallot, however (Fig. 2), the interventricular groove rotates to the left while the transition zone between $\mathrm{qR}$ (or rsR) and $\mathrm{rS}$ complexes moves to the right. Consideration of this diagram suggests that the rS pattern recorded from V2-V5 is in fact derived from the underlying right ventricular epicardium, and that the rsR complexes recorded in leads V3R-V8R may arise chiefly from the left ventricular surface. This implies that if direct leads could be recorded from the right and left ventricular walls they would not demonstrate " electrical predominance" of the right ventricle, but would show $\mathrm{rS}$ and $\mathrm{qR}$ patterns respectively as in health. The investigation described below is an attempt to determine whether this is in fact the case. 


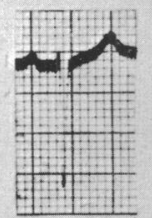

I

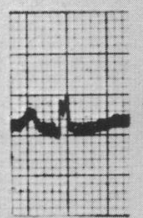

III

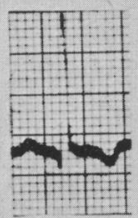

III

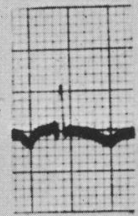

OVR

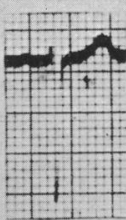

OVL

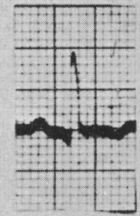

QVF

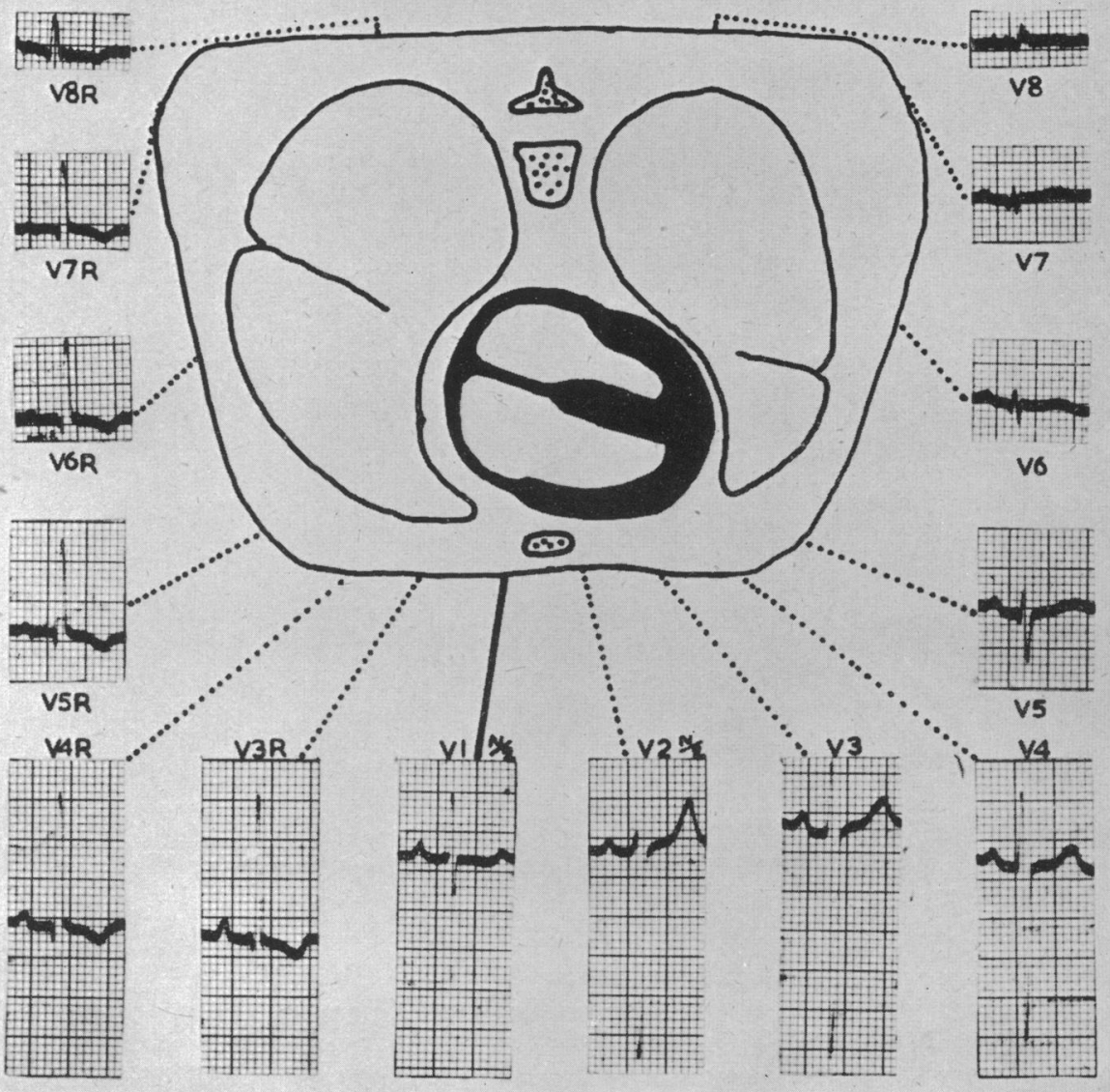

Fig. 2.-Diagram and electrocardiograms from man, aged 19 (V.B.), with tetralogy of Fallot. A similar diagram to Fig. 1. The entire anterior surface of the heart now consists of right ventricular wall. Half normal sensitivity is used in recording leads V1 and V2. The electrograms of this patient are shown in Fig. 5.

\section{INVESTIGATION}

Cases have been selected that were about to undergo thoracotomy. Except where otherwise stated thoracotomy has been performed via a left lateral incision. In this way a small area of the left cardiac surface was exposed. The pericardium was as a rule not incised, but with one exception the descending branch of the left coronary artery could be identified and this was considered to mark approximately the boundary zone between the two ventricles. An exploring electrode consisting of an insulated wire terminating in a moist cotton wool swab of about half an inch diameter was laid on the exposed pericardium. If it contacted other thoracic structures as well this is indicated. 


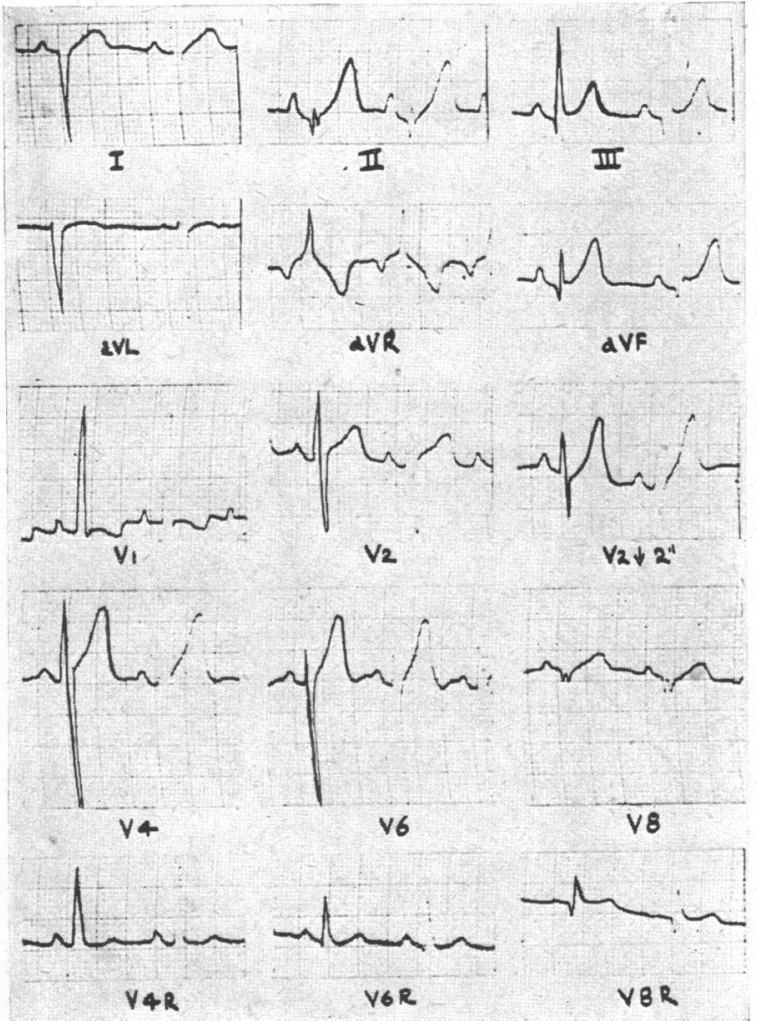

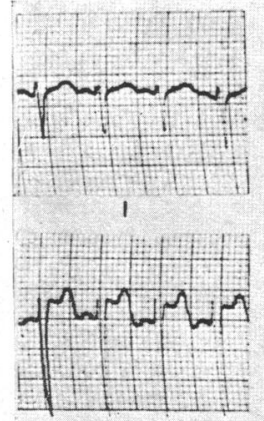

3

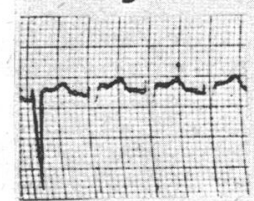

6

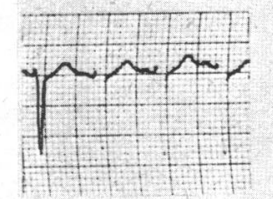

I

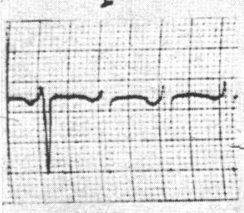

VL

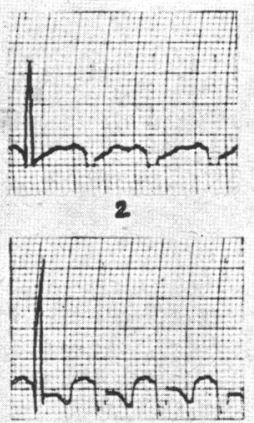

4

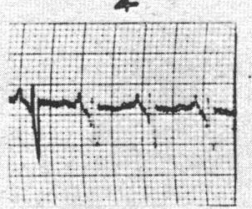

7

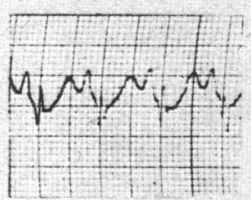

II

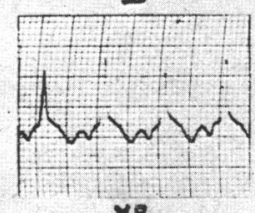

VR

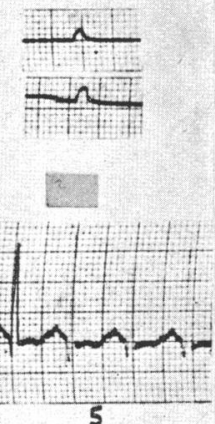

5

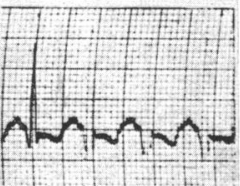

8

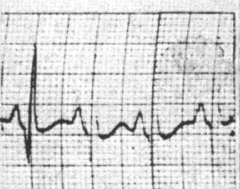

III

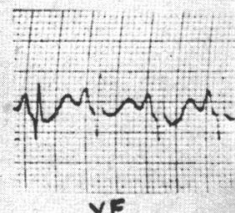

VF

FIG. 3.-Electrocardiogram for a boy (A. T.), aged 4, with the tetralogy of Fallot. On the left is the pre-operative electrocardiogram. The electrograms (on the right) are recorded from the heart as follows:-

$1,3=$ Anterior wall of right ventricle.

$6=$ Pulmonary conus.

$2,4=$ Lateral wall of left ventricle.

$5=$ Posterior wall of left ventricle near diaphragm.

$7=$ High posterior wall of left ventricle near auriculo-ventricular groove.

$8=$ Posterior wall of left ventricle between 5 and 7 .

Graphs 5,6 and 8 are impure electrograms as the electrode was also in contact with the left lung. One complex of each tracing has been touched up for purposes of reproduction. The indifferent electrode was attached in this case to the right arm. Standardization varied between the limits shown. Below, on the right, are the limb leads recorded during the operation.

The unipolar indifferent electrode of Wilson was used in all cases except that illustrated in Fig. 3, where the right arm was used as an indifferent electrode. Unless otherwise indicated normal standardization is used. On the left side of Fig. 1 is an example of the qR and rS complexes normally recorded from the left and right ventricles respectively in health.

Ten cases of tetralogy of Fallot were investigated. All showed the electrocardiogram considered typical of right ventricular hypertrophy and all were clinically typical of this condition. In most cases the diagnosis was confirmed by means of cardiac catheterization and angiocardiography.

\section{RESULTS}

In the first case, illustrated in Fig. 3, there was a good exposure of both ventricles, and records were obtained from several points on each. The septal region in this case was avoided. It will be 
seen that $\mathrm{rS}$ complexes were recorded from the right ventricle. $\mathrm{qR}$ complexes were recorded from the left, except when the electrode lay near the auriculo-ventricular groove posteriorly (graph 7, Fig. 3). An identical case is not illustrated. In two similar cases (one illustrated in Fig. 4) the initial $r$ wave over the right ventricular surface was replaced by a $q$ wave in the region of the septum.

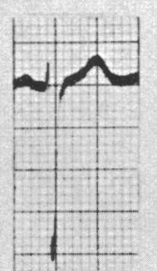

I

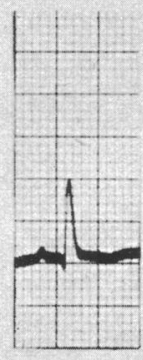

VER

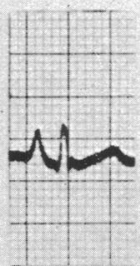

ㅍ

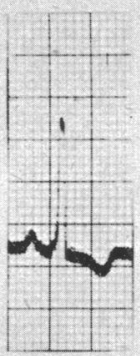

VSR

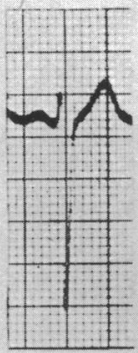

RVEI

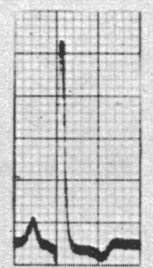

III

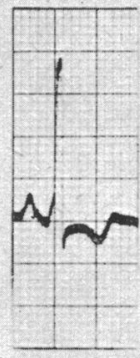

V

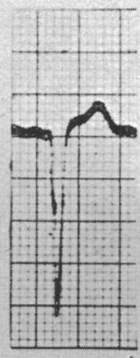

RVE2
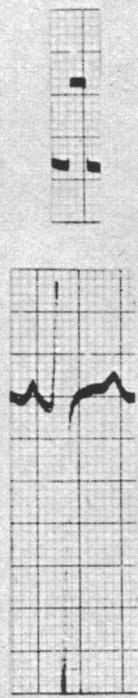

V2

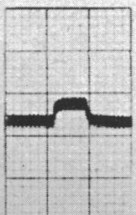

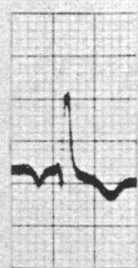

QVR

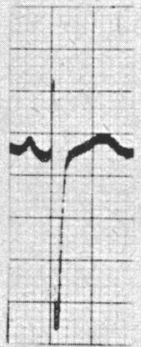

V4

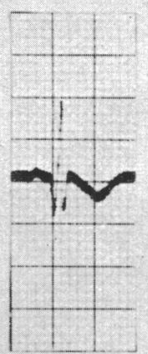

LVEI

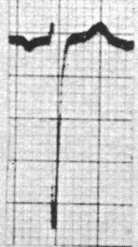

aVL

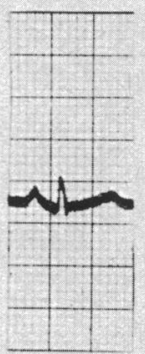

Vo

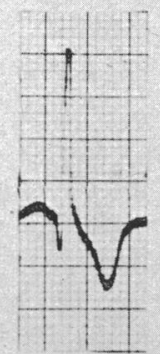

LVE 2

Fig. 4.-Electrocardiogram from a woman (S.G.), aged 18 years, with tetralogy of Fallot. Electrograms with their standardization shown below as follows:-

R.V.E. 1 = Anterior wall of right ventricle approximately two inches from descending branch of left coronary artery.

R.V.E. 2 = Anterior wall of right ventricle approximately half an inch from same artery.

L.V.E. 1 = Left lateral wall of left ventricle approximately half an inch from the same artery.

L.V.E. 2 = Posterolateral wall of left ventricle approximately one and a half inches from same artery.

In Fig. 5 are shown the electrograms of the patient whose electrocardiogram was illustrated in Fig. 2. In this case very little of the right ventricle was available for exploration and possibly for this reason an intrinsic $r$ wave could not be demonstrated. A large area of the left ventricle was shown to transmit a $\mathrm{qR}$ pattern. In the case illustrated in Fig. 6 the two ventricles could not be identified with certainty. Leads from the anterolateral surface of the heart both before and after the completion of the anastomosis showed an rS pattern. After the anastomosis, however, a qS complex was obtained from the postero-lateral surface of the heart and a $\mathrm{qR}$ pattern was not recorded until the electrode was thrust round to the back of the heart where it was in contact with both the 


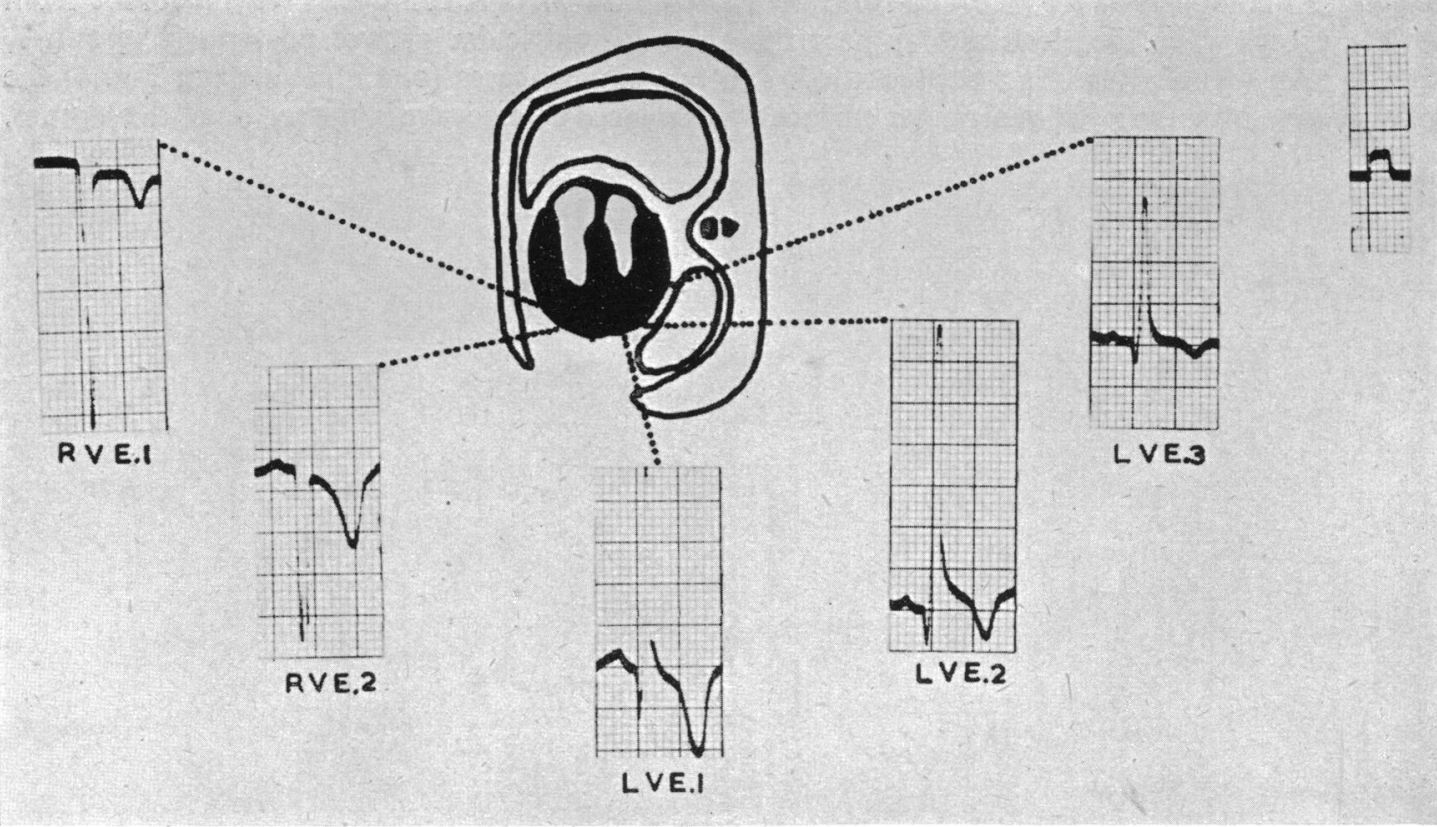

FIG. 5.-Electrogram of case illustrated in Fig. 2. T.V.E.1 in this case was not more than one inch from the descending branch of the left coronary artery.

lung and the pericardium. It is probable that in this case a degree of rotation of the heart greater than usual had taken place and that during the course of the anastamosis a further change in position had taken place leaving only the right ventricle exposed.

Although the ventricles in two other cases could be defined, adequate exploration of the left ventricle could not be performed. In one an $\mathrm{rS}$ pattern and in the other a qS pattern was recorded from the right ventricular surface. In the latter case an anterior approach was employed and procaine was injected into the wall of the right ventricle; records were taken after the performance of a pulmonary valvulotomy (Brock, 1949) through the right ventricular wall. Although records were obtained from the area one inch to the left of the descending branch of the left coronary artery qS complexes only were obtained; the electrical transition point may therefore be at least one inch to the left of this vessel.

The last two cases to be described were similar and one only is illustrated (Fig. 7). Both were characteristic in that the left lateral wall of the right and left ventricles transmitted predominantly negative (rS) and positive (qR) patterns respectively. However, when the exploring electrode was thrust deeply into the potential space between the heart and the anterior chest wall in the region of the sternum a predominantly positive complex was recorded (curve E1, Fig. 7). The exact position of the electrode was uncertain, but was in the region of the auriculo-ventricular groove near the posterior surface of the sternum; the absence of a typical large auricular $\mathbf{P}$ wave suggests that it was not in contact with the right auricle. It must also be remembered that the electrode was in as good contact with the chest wall as the pericardium in this position, and consequently cannot be regarded as a pure electrogram. The origin of this predominantly positive pattern is therefore uncertain.

\section{Discussion}

In the ten cases investigated most, if not all, of the right ventricular surface transmits, as in health, an $\mathrm{rS}$ pattern, which is similar to that derived from the immediately overlying left præcordium. 


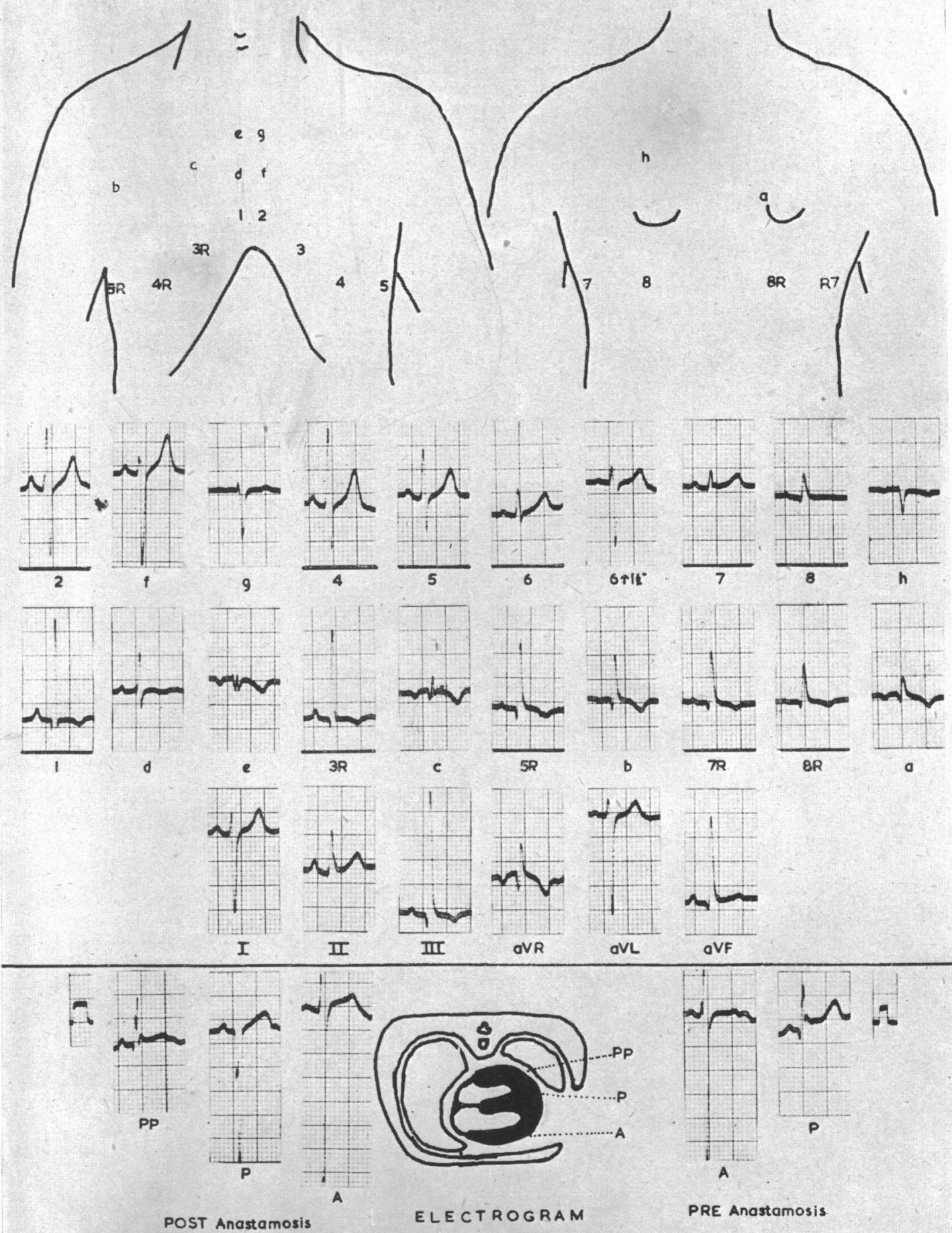

FIG. 6.-Diagram and electrograms from boy (C.S.), aged 16 years, with the tetralogy of Fallot. The diagrams above show the approximate positions of the chest electrode for each record. V leads used throughout. The electrograms below on the right were taken before, and the left after the anastomosis. The descending branch of the left coronary artery was not identified. The lower diagram shows the approximate sites on the lateral surface of the heart from which the electrograms were recorded. When the electrode was in position PP it was in contact with the lung as well as the heart. 


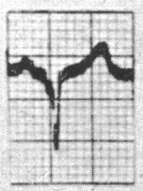

I

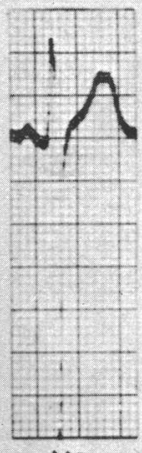

V2

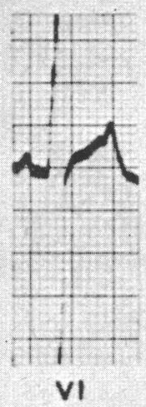

vi

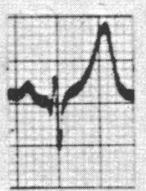

III

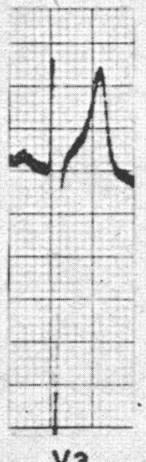

v3
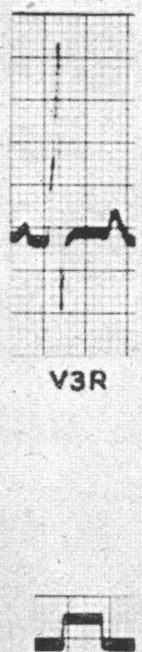

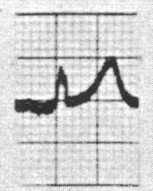

III
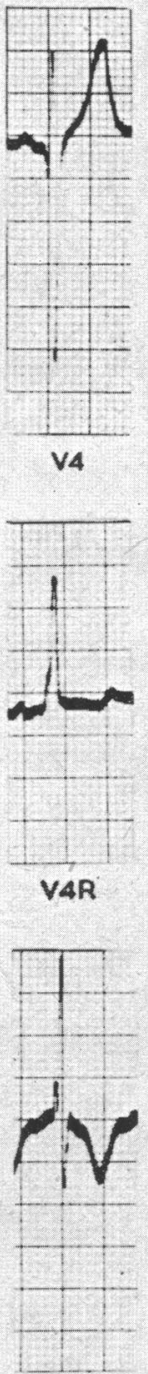

E.I.
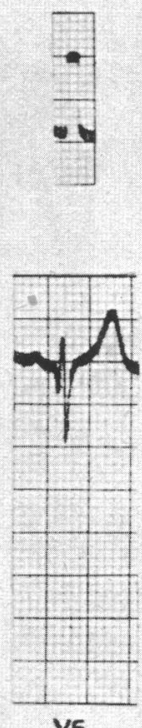

v5

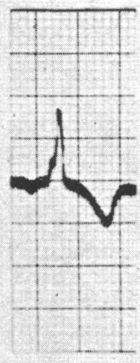

V5R

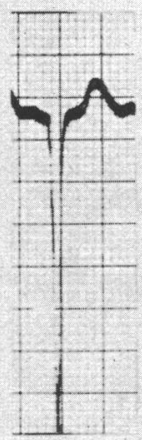

RVE

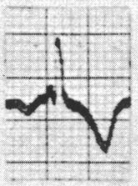

aVR

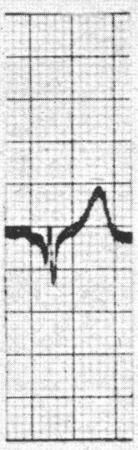

vo
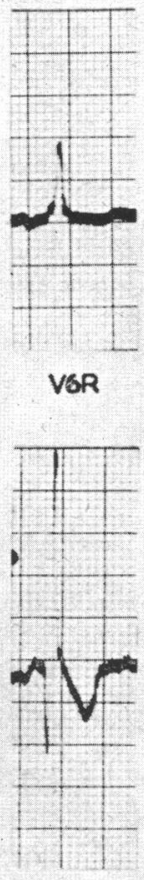

LVE

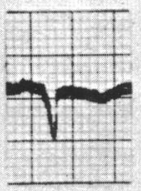

oVL
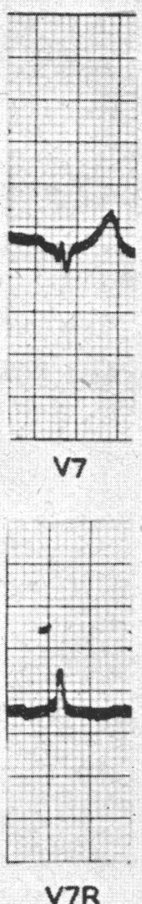

V7R
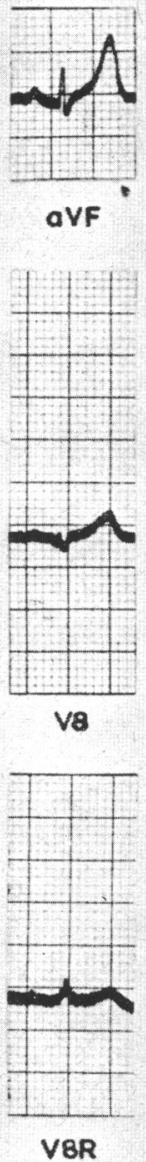

VBR

Fig. 7.-Electrocardiograms for a boy (E.S.), aged 12 years, with the tetralogy of Fallot.

R.V.E. = Right ventricular electrogram approximately 1 inch from descending branch of left coronary artery. L.V.E. = Left ventricular electrogram.

E.1. = Retrosternal area. (See text). 
Similarly, a qR pattern may be recorded from the left ventricular surface. The origin of the upright complexes derived from the right chest in these cases is uncertain.

It is possible that an area of the right ventricle in the region of the auriculo-ventricular groove transmits an $r R$ pattern. An alternative explanation is that the large $R$ wave found over the right chest is in fact derived from the left ventricular surface as a result of the rotation of the heart. Kossman et al. (1948) suggested that the pattern of right ventricular hypertrophy in a case of cor pulmonale which they studied was due to rotation of the heart with almost complete reversal of the electrical fields of the two ventricles in the thorax, and there are some grounds for thinking that this explanation may hold here.

\section{SUMMARY}

The electrocardiographic pattern considered typical of hypertrophy of the right ventricle is described.

Direct cardiac leads have been recorded at operation in ten cases of tetralogy of Fallot with this typical pattern.

The pattern derived from the left ventricle is found to be predominantly positive and that from the right predominantly negative, as in health.

It is concluded that the rS pattern found over the left pracordium in these cases is derived from the underlying right ventricular epicardium as a result of the rotation of the heart.

The origin of the large $\mathrm{R}$ wave found over the right chest is uncertain, but it may be derived from the left ventricular surface also as a result of rotation.

I wish to record my sincere thanks to Dr. J. B. Lowe who has given up much of his time to assist me during the investigations described here. I am also deeply indebted to Mr. W. Cleland, Mr. T. Holmes Sellors, Mr. R. C. Brock, Mr. G. C. Laurie Pile and Mr. L. Fatti for most kindly allowing me to record electrograms during their operations, and to Dr. B. van Lingen and Professor G. A. Elliot for their helpful suggestions in the writing of this paper.

My deepest gratitude is due to Dr. Paul Wood for his continuous stimulation and encouragement.

\section{REFERENCES}

Brock, R. C. (1949). Brit. med. J., $2,399$.

Goldberger, E. (1944). Amer. Heart J., 28, 621.

Kossman, C. E., Berger, A. R., Brumlik, J., and Briller, S. (1948). Ibid., 35, 309.

Myers, G. B., Klein, H. A., and Stofer, B. E. (1948). Ibid., 35, 1.

Sokolow, M., Lyon, T. P. (1949). Ibid., 38, 273.

Wilson, F. M., Berger, A. R., Brumlik, J., and Briller, S. (1944). Ibid.: $27,19$. 